\title{
Sensitive $\mathrm{Cu}^{2+-} \mathrm{Cu}^{2+}$ Distance Measurements in a Protein-DNA Complex by Double-Quantum Coherence ESR
}

\author{
Sharon Ruthstein ${ }^{+, \&, 1}$, Ming $\mathrm{Ji}^{+}, \&$, Preeti Mehta ${ }^{\wedge}, 2$, Linda Jen-Jacobson ${ }^{\wedge}$, and Sunil \\ Saxena ${ }^{\&, *}$ \\ \&Department of Chemistry, University of Pittsburgh, Pittsburgh, PA, 15260 \\ ^Department of Biological Sciences, University of Pittsburgh, Pittsburgh, PA, 15260
}

\section{Abstract}

Double Quantum Coherence (DQC) ESR spectroscopy is applied to measure the $\mathrm{Cu}^{2+}-\mathrm{Cu}^{2+}$ distance in the EcoRI-DNA complex. A simple method is proposed to reduce the contribution of nuclear hyperfine and quadrupole interactions to such data. The effects of such interactions between the electron spin of $\mathrm{Cu}^{2+}$ and neighboring nuclei on the DQC data, make it difficult to measure the nanometer range interspin distance. The DQC data is in good agreement with results obtained by Double Electron Electron Resonance (DEER) spectroscopy. At the same time, the signal to noise ratio per shot in DQC is high. Taken together, these results provide impetus for further development of paramagnetic metal ion-based DQC techniques.

\section{Keywords}

ESR Spectroscopy; DQC; EcoRI; electron-nuclear interactions; electron-electron dipolar interaction

\section{Introduction}

Herein, we demonstrate a simple way to measure paramagnetic metal ion based nanoscale distances in proteins. The advent of Double Electron Electron Resonance (DEER) $)^{1,2}$ and Double Quantum Coherence (DQC) $)^{3,4}$ methods that measure nanometer range interspin distances have had a profound impact on the application of ESR in biological research. ${ }^{5-12}$ Most of these distance measurements are based on the methanethiolsulfonate nitroxide spinlabel, which is chemically attached to the cysteine residue in proteins. ${ }^{13}$ The extension of DEER and DQC distance measurements to paramagnetic metal ions can potentially generalize the technique to many proteins that contain endogenous metal ion binding sites. However, DEER and DQC with metal ions as spin probe are challenging because of large ESR spectral width and low signal to noise ratio (SNR). Nevertheless, our group ${ }^{14,15}$ and others ${ }^{16-26}$ have made tangible progress. In principle DQC can have a high SNR per shot. This provides impetus for further research of DQC. On the other hand, the DQC signal has substantial contributions from electron-nuclear interactions between the electron spin and neighboring ${ }^{14} \mathrm{~N}$ as well as ${ }^{1} \mathrm{H}$ nuclear spins that are present in the amino-acid coordination

\footnotetext{
*To whom correspondence should be addressed. Phone: (412) 624-8680. Fax: (412) 624-8611. sksaxena@ pitt.edu..

${ }^{+}$S.R. and M.J. contributed equally to this work.

${ }^{1}$ Current address: Department of Chemistry, Faculty of Exact Science, Bar Ilan University, Ramat-Gan, Israel, 5290002

${ }^{2}$ Current address: Department of Biochemistry, Stony Brook University, Stony Brook, NY 11794
}

Supporting Information. Data processing and DQC simulation, details of DEER experiment, and signal to noise ratio calculation. This material is available free of charge via the Internet at http://pubs.acs.org. 
environment. These nuclear modulation ${ }^{27}$ effects swamp the modulation due to the electronelectron dipolar (EED) interactions and make it difficult to measure the interspin distance.

EcoRI is a $62 \mathrm{kDa}$ homodimeric protein ${ }^{28,29}$ that recognizes and binds to the 5 'GAATTC-3'DNA sequence with high specificity ${ }^{30-32}$, even in the absence of metal cofactors. In the presence of $\mathrm{Mg}^{2+}$, EcoRI catalyzes cleavage of both DNA strands at this site. When $\mathrm{Mg}^{2+}$ is replaced by other metal ions the cleavage rates decrease according to the series $\mathrm{Mg}^{2+} \approx \mathrm{Mn}^{2+}>\mathrm{Co}^{2+}>>\mathrm{Zn}^{2+}>>\mathrm{Cd}^{2+}>\mathrm{Ni}^{2+} .{ }^{31}$ Interestingly, $\mathrm{Cu}^{2+}$ is a powerful inhibitor of EcoRI catalysis. ${ }^{33}$ In order to shed light on the molecular mechanism of catalytic inhibition by $\mathrm{Cu}^{2+}$, we have recently exploited DEER based distance measurements to determine that $\mathrm{Cu}^{2+}$ binds to His114 in each subunit of EcoRI, ${ }^{33}$ at points $13 \AA$ from the $\mathrm{Mg}^{2+}$ positions in the catalytic sites. The positions of the His114 sidechains are consequently altered, and the resulting disruption of critical protein-DNA interactions and water molecules in the catalytic centers leads to inhibition of catalysis. ${ }^{33}$

In this work we present a simple way to minimize the low frequency nuclear peaks in the DQC spectrum and resolve the dipolar interaction between two paramagnetic probes $\mathrm{Cu}^{2+}$ with high sensitivity in the complex of restriction endonuclease EcoRI with its cognate DNA.

\section{Experimental methods}

\section{Enzyme expression and purification}

The EcoRI protein was expressed from a maltose-binding protein-EcoRI (MBP-EcoRI) fusion construct. Details for generation of the fusion gene and expression of the fusion protein are given in supporting information for reference 33. The complete EcoRI protein without extra amino acids was isolated, purified, and characterized as described in reference 34.

\section{$\mathrm{Cu}^{2+-}$-EcoRI-DNA sample preparation}

A solution of EcoRI $(5 \mu \mathrm{M})$ in the presence of fivefold molar excess of TCGCGAATTCGCG was exchanged into $30 \mathrm{mM}$ N-ethylmorpholine (NEM) buffer, which contains $0.3 \mathrm{M} \mathrm{NH}_{4} \mathrm{Cl}, 10 \%$ dioxane, $30 \%$ deuterated glycerol $(\mathrm{d} 8), 65 \% \mathrm{D}_{2} \mathrm{O}(\mathrm{pH} 8.0)$ and concentrated. The final concentrations of EcoRI and DNA were $380 \mu \mathrm{M}$ and $1.5 \mathrm{mM}$, respectively. Isotopically enriched ${ }^{63} \mathrm{CuCl}_{2}$ (Cambridge Isotope Labs, Inc) was added at a 4:1 molar ratio $\left(\mathrm{Cu}^{2+}\right.$ : protein dimer). The sample was stored at $-80^{\circ} \mathrm{C}$ and flash-frozen before each ESR experiment.

\section{Electron Spin Resonance Spectroscopy}

All of the pulsed ESR experiments were performed on a Bruker Elexsys 580 spectrometer at $20 \mathrm{~K}$, with a MD5 resonator. The six pulse DQC-ESR was using a $\pi / 2-\mathrm{t}_{\mathrm{p}}+\mathrm{dt}-\pi-\mathrm{t}_{\mathrm{p}}+\mathrm{dt}-\pi / 2-\mathrm{t}_{1}-$ $\pi-t_{1}-\pi / 2-t_{2}-\pi$-echo sequence. A 64 step phase cycle was carried out to select the correct coherence pathway and the DQC-echo after the sixth pulse was integrated. ${ }^{14,35}$ The $\pi / 2$ pulse and $\pi$ pulse lengths were $8 \mathrm{~ns}$ (or $12 \mathrm{~ns}, 16 \mathrm{~ns}$ and $20 \mathrm{~ns}$ ) and $16 \mathrm{~ns}$ (or $24 \mathrm{~ns}, 32 \mathrm{~ns}$ and $40 \mathrm{~ns}$ ), respectively. The interval $t_{p}$ was incremented from $80 \mathrm{~ns}$ with a stepsize of $8 \mathrm{~ns}$, for a total of 360 points. The interval $t_{1}$ was $80 \mathrm{~ns}$ and $\mathrm{t}_{2}$ was $200 \mathrm{~ns}$. The shot repetition time was $5 \mathrm{~ms}$ and all the DQC time domain data were averaged for 24 scans. Each DQC experiment was done at $3350 \mathrm{G}$ where the maximum echo signal was observed. Details of the DEER experiment are given in supporting information and reference 33. 


\section{Results and Discussion}

Figure 1 shows the $\mathrm{Cu}^{2+}{ }_{-}$DQC data obtained at the $g \perp$ position of the $\mathrm{Cu}^{2+}$ ESR absorption spectrum. The lengths of the $\pi$ pulse were 16 ns (black solid line) and $40 \mathrm{~ns}$ (grey solid line), respectively.

The two spectra in Figure 1b with different length of $\pi$ pulse clearly contain peaks that can be attributed to the ${ }^{14} \mathrm{~N}$-nuclear modulations in the $0-5 \mathrm{MHz}$ region ${ }^{14}$ as well as the ${ }^{1} \mathrm{H}$ nuclear peaks at $\sim 14 \mathrm{MHz}$. For the purpose of nanoscale distance measurements the 0-5 $\mathrm{MHz}{ }^{14} \mathrm{~N}$-nuclear peaks are particularly problematic, since the EED interaction is in this region. Interestingly, the intensity of the peak at $\sim 1.46 \mathrm{MHz}$ in the DQC spectrum (Figure 1b) with $40 \mathrm{~ns} \pi$ pulse is much lower compared to the spectrum with $16 \mathrm{~ns} \pi$ pulse. With shorter $\pi$ pulse, e. g., $16 \mathrm{~ns}$, the spectral excitation probability of the DQC EED interaction is about $0.16 .{ }^{35}$ With the longer $\pi$ pulse of $40 \mathrm{~ns}$, the spectral excitation probability of the DQC EED interaction is about $0.04 .{ }^{35}$ It is very clear that longer $\pi$ pulse cannot substantially populate the double quantum coherence of EED interaction. However, the nuclear hyperfine and quadrupole transitions are weakly sensitive to the length of $\pi$ pulse. ${ }^{27}$ This suggests that $1.46 \mathrm{MHz}$ peak might originate from the $\mathrm{Cu}^{2+}$-EED interaction.

Since the experimental DQC signal is a multiplication of the EED and the nuclear signals, division of the $\pi=16 \mathrm{~ns}$ DQC data by the $\pi=40 \mathrm{~ns}$ DQC data may suppress the nuclear signals and resolve the EED signal. ${ }^{14,35}$ Figure 2a shows the comparison of the DQC time domain data, after dividing the $\pi=16 \mathrm{~ns}$ signal by the $\pi=40 \mathrm{~ns}$ signal (black solid line), with the DEER data after subtraction of the homogeneous background (grey solid line). ${ }^{33}$ The modulation periods of the two time domain traces are comparable. In this analysis, the DQC signal with $40 \mathrm{~ns} \pi$ pulse was empirically chosen for division - the data with other choices are shown in the Supporting Information. The data acquisition time of DQC (including two traces) and DEER are $\sim 50 \mathrm{hrs}$ and $\sim 43 \mathrm{hrs,} \mathrm{respectively.} \mathrm{The} \mathrm{data} \mathrm{collection} \mathrm{time} \mathrm{in} \mathrm{DQC}$ can be reduced by implementation of on-board phase cycling.

The DQC spectrum after division, shown in Figure $2 \mathrm{~b}$ (black solid line), clearly resolves the major peak at $\sim 1.46 \mathrm{MHz}$, which is consistent with the DEER data (Figure $2 \mathrm{~b}$, grey solid line). Nevertheless, the DQC spectrum is narrower than the DEER spectrum. In the experimental DQC data, there might be some small residual peaks from electron- ${ }^{14} \mathrm{~N}$ interactions that occur at $\sim 3 \mathrm{MHz}$.

Figure 2 also shows the simulated DQC data (black dotted line). Based on the best fitting of the DQC time domain data shown in Figure 2, we obtained a most probable $\mathrm{Cu}^{2+}-\mathrm{Cu}^{2+}$ distance of $\sim 35 \AA$ with a standard deviation of $1 \AA$ (Figure 2b, black solid line inset). This result is consistent with our observation from DEER experiments (Figure $2 b$, red dotted line inset). The simulated DQC spectrum is in accord with the dominant peak obtained in the experiment, although the DQC data is narrower. ${ }^{33}$ Details of the simulations are provided in the Supporting Information. It is likely that in DQC a narrower range of orientations is selected because the microwave pulses have a finite coverage. Despite this, the signal to noise ratio per shot in DQC is high (cf. figure caption in Figure 2 and Supporting Information).

\section{Conclusions}

We have clearly shown here that metal ion based nanometer range distance can be measured by DQC. DEER has a significant advantage due to the ease of implementation on commercial instrument. Nevertheless, the DQC data have high SNR per shot. Further improvements can be made by the use of narrower pulse lengths ${ }^{35}$ and by understanding the role of orientational effects in such DQC measurements. ${ }^{15,36}$ 


\section{Supplementary Material}

Refer to Web version on PubMed Central for supplementary material.

\section{Acknowledgments}

This work was supported by a National Science Foundation (MCB 1157712) grant. Protein expression was supported by NIH MERIT (5R37-GM02907) grant to L.J.-J.

\section{REFERENCES}

(1). Milov AD, Ponomarev AB, Tsvetkov YD. Electron-Electron Double Resonance in Electron Spin Echo: Model Biradical Systems and the Sensitized Photolysis of Decalin. Chem. Phys. Lett. 1984; 110:67-72.

(2). Pannier M, Viet S, Godt A, Jeschke G, Spiess HW. Dead-Time Free Measurement of DipoleDipole Interactions between Electron Spins. J. Magn. Reson. 2000; 142:331-340. [PubMed: 10648151]

(3). Borbat PP, Freed JH. Multiple-Quantum ESR and Distance Measurements. Chem. Phys. Lett. 1999; 313:145-154.

(4). Freed JH. New Technologies in Electron Spin Resonance. Annu. Rev. Phys. Chem. 2000; 51:655689. [PubMed: 11031296]

(5). Park S-Y, Borbat PP, Gonzalez-Bonet G, Bhatnagar J, Pollard AM, Freed JH, Bilwes AM, Crane BR. Reconstruction of the Chemotaxis Receptor-Kinase Assembly. Nat. Struct. Mol. Bio. 2006; 13:400-407. [PubMed: 16622408]

(6). Altenbach C, Kusnetzow AK, Ernst OP, Hofmann KP, Hubbell WL. High-Resolution Distance Mapping in Rhodopsin Reveals the Pattern of Helix Movement Due to Activation. Proc. Natl. Acad. Sci. USA. 2008; 105:7439-7444. [PubMed: 18490656]

(7). Stone KM, Townsend JE, Sarver J, Sapienza PJ, Saxena S, Jen-Jacobson L. Electron Spin Resonance Shows Common Structural Features for Different Classes of EcoRI-DNA Complexes. Angew. Chem. Int. Ed. 2008; 47:10192-10194.

(8). Bleicken S, Classen M, Padmavathi PVL, Ishikawa T, Zeth K, Steinhoff H-J, Bordignon E. Protein Structure and Folding. J. Biol. Chem. 2010; 285:6636-6647. [PubMed: 20008353]

(9). Dockter C, Volkov A, Bauer C, Polyhach Y, Joly-Lopez Z, Jeschke G, Paulsen H. Refolding of the Integral Membrane Protein Light-Harvesting Complex II Monitored by Pulse EPR. Proc. Natl. Acad. Sci. USA. 2009; 106:18485-18490. [PubMed: 19833872]

(10). Kear JL, Blackburn ME, Veloro AM, Dunn BM, Fanucci GE. Subtype Polymorphisms Among HIV-1 Protease Variants Confer Altered Flap Conformations and Flexibility. J. Am. Chem. Soc. 2009; 131:14650-14651. [PubMed: 19788299]

(11). Sugata K, Song L, Nakamura M, Ueki S, Fajer PG, Arata T. Nucleotide-Induced Flexibility Change in Neck Linkers of Dimeric Kinesin as Detected by Distance Measurements Using SpinLabeling EPR. J. Mol. Biol. 2009; 386:626-636. [PubMed: 19154743]

(12). Zou P, Bortolus M, Mchaourab HS. Conformational Cycle of the ABC Transporter MsbA in Liposomes: Detailed Analysis Using Double Electron-Electron Resonance Spectroscopy. J. Mol. Biol. 2009; 393:586-597. [PubMed: 19715702]

(13). Hubbell WL, Altenbach C. Investigation of Structure and Dynamics in Membrane Proteins Using Site-Directed Spin Labeling. Curr. Opin. Sctruct. Biol. 1994; 4:566-573.

(14). Becker JS, Saxena S. Double Quantum Coherence Electron Spin Resonance on Coupled Cu(II)Cu(II) Electron Spins. Chem. Phys. Lett. 2005; 414:248-252.

(15). Yang Z, Kise D, Saxena S. An Approach towards the Measurement of Nanometer Range Distances Based on $\mathrm{Cu}^{2+}$ Ions and ESR. J. Phys. Chem. B. 2010; 114:6165-6174. [PubMed: 20397677]

(16). Elsässer C, Brecht M, Bittl R. Pulsed Electron-Electron Double Resonance on Multinuclear Metal Clusters: Assignment of Spin Projection Factors Based on the Dipolar Interaction. J. Am. Chem. Soc. 2002; 124:12606-12611. [PubMed: 12381206] 
(17). Narr E, Godt A, Jeschke G. Selective Measurements of a Nitroxide-Nitroxide Separation of $5 \mathrm{~nm}$ and a Nitroxide-Copper Separation of $2.5 \mathrm{~nm}$ in a Terpyridine-Based Copper(II) Complex by Pulse EPR Spectroscopy. Angew. Chem. Int. Ed. 2002; 41:3907-3910.

(18). Amsterdam, I. M. C. v.; Ubbink, M.; Canters, GW.; Huber, M. Measurement of a Cu-Cu Distance of $26 \AA$ by a Pulsed EPR Method. Angew. Chem. Int. Ed. 2003; 42:62-64.

(19). Kay CWM, Mkami HE, Cammack R, Evans RW. Pulsed ELDOR Determination of the Intramolecular Distance between the Metal Binding Sites in Dicupric Human Serum Transferrin and Lactoferrin. J. Am. Chem. Soc. 2007; 129:4868-4869. [PubMed: 17394319]

(20). Bode BE, Plackmeyer J, Prisner TF, Schiemann O. PELDOR Measurements on a NitroxideLabeled Cu(II) Porphyrin: Orientation Selection, Spin-Density Distribution, and Conformational Flexibility. J. Phys. Chem. A. 2008; 112:5064-5073. [PubMed: 18491846]

(21). Raitsimring AM, Gunanathan C, Potapov A, Efremenko I, Martin JML, Milstein D, Goldfarb D. $\mathrm{Gd}^{3+}$ Complexes as Potential Spin Labels for High Field Pulsed EPR Distance Measurements. J. Am. Chem. Soc. 2007; 129:14138-14140. [PubMed: 17963387]

(22). Potapov A, Song Y, Meade TJ, Goldfarb D, Astashkin AV, Raitsimring A. Distance Measurements in Model Bis-Gd(III) Complexes with Flexible "Bridge". Emulation of Biological Molecules Having Flexible Structure with Gd(III) Labels Attached. J. Magn. Reson. 2010; 205:38-49. [PubMed: 20418132]

(23). Potapov A, Yagi H, Huber T, Jergic S, Dixon NE, Otting G, Goldfarb D. Nanometer-Scale Distance Measurements in Proteins Using $\mathrm{Gd}^{3+}$ Spin Labeling. J. Am. Chem. Soc. 2010; 132:9040-9048. [PubMed: 20536233]

(24). Gordon-Grossman M, Kaminker I, Gofman Y, Shai Y, Goldfarb D. W-Band Pulse EPR Distance Measurements in Peptides Using Gd $^{3+}$-Dipicolinic Acid Derivatives as Spin Labels. Phys. Chem. Chem. Phys. 2011; 13:10771-10780. [PubMed: 21552622]

(25). Yagi H, Banerjee D, Graham B, Huber T, Goldfarb D, Otting G. Gadolinium Tagging for HighPrecision Measurements of $6 \mathrm{~nm}$ Distances in Protein Assemblies by EPR. J. Am. Chem. Soc. 2011; 133:10418-10421. [PubMed: 21661728]

(26). Banerjee D, Yagi H, Huber T, Otting G, Goldfarb D. Nanometer-Range Distance Measurement in a Protein Using $\mathrm{Mn}^{2+}$ Tags. J. Phys. Chem. Lett. 2012; 3:157-160.

(27). Schweiger, A.; Jeschke, G. Principles of Pulse Electron Paramagnetic Resonance. Oxford University Press; Oxford: 2001.

(28). Kim YC, Grable JC, Love R, Greene PJ, Rosenberg JM. Refinement of Eco RI Endonuclease Crystal Structure: a Revised Protein Chain Tracing. Science. 1990; 249:1307-1309. [PubMed: 2399465]

(29). Grigorescu, A.; Horvath, M.; Wilkosz, PA.; Chandrasekhar, K.; Rosenberg, JM. Restriction Endonucleases. Springer-Verlag; Heidelberg: 2004.

(30). Lesser DR, Kurpiewski MR, Jen-Jacobson L. The Energetic Basis of Specificity in the Eco RI Endonuclease--DNA Interaction. Science. 1990; 250:776-786. [PubMed: 2237428]

(31). Vipond IB, Baldwin GS, Halford SE. Divalent Metal Ions at the Active Sites of the EcoRV and EcoRI Restriction Endonucleases. Biochemistry. 1995; 34:697-704. [PubMed: 7819265]

(32). Pingoud A, Fuxreiter M, Pingoud V, Wende W. Type II Restriction Endonucleases: Structure and Mechanism. Cell. Mol. Life Sci. 2005; 62:685-707. [PubMed: 15770420]

(33). Yang Z, Kurpiewski MR, Ji M, Townsend JE, Mehta P, Jen-Jacobson L, Saxena S. ESR Spectroscopy Identifies Inhibitory $\mathrm{Cu}^{2+}$ Sites in a DNA-Modifying Enzyme to Reveal Determinants of Catalytic Specificity. Proc. Natl. Acad. Sci. USA. 2012; 109:E993-E1000. [PubMed: 22493217]

(34). Dylla-Spears R, Townsend JE, Sohn LL, Jen-Jacobson L, Muller SJ. Fluorescent Marker for Direct Detection of Specific dsDNA Sequences. Anal. Chem. 2009; 81:10049-10054. [PubMed: 19908852]

(35). Borbat, PP.; Freed, JH. Distance Measurements in Biological Systems by EPR. Vol. Vol. 19. Kluwer Academic/Plenum Publisher; New York: 2000. Double-Quantum ESR and Distance Measurements. Chapter 9 
(36). Misra SK, Borbat PP, Freed JH. Calculation of Double-Quantum-Coherence Two-Dimensional Spectra: Distance Measurements and Orientational Correlations. Appl. Magn. Reson. 2009; 36:237-258. [PubMed: 20161423] 


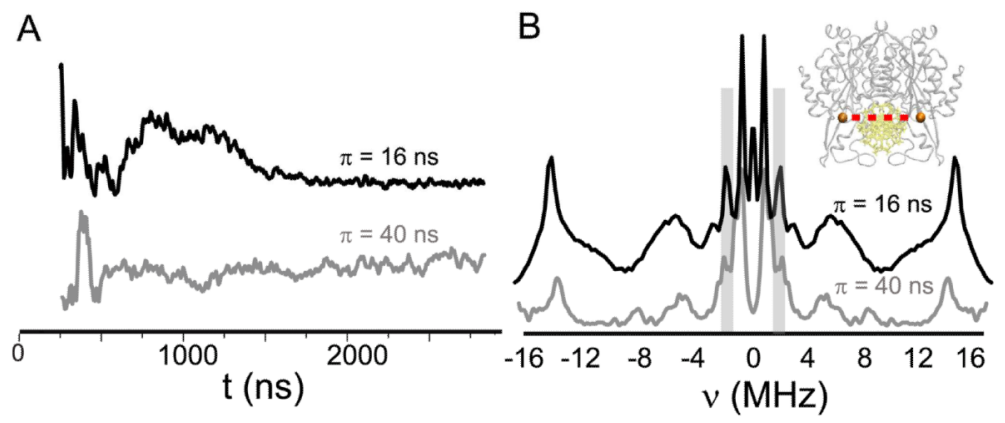

Figure 1.

The experimental $\mathrm{Cu}^{2+}$-DQC (a) time domain signals and (b) spectra of the EcoRI-DNA complex, measured at two different $\pi$ pulse lengths: $\pi=16$ ns (black solid line) and $\pi=40 \mathrm{~ns}$ (grey solid line). The signal to noise ratios for the two traces with $\pi=16 \mathrm{~ns}$ and $\pi=40 \mathrm{~ns}$ are $\sim 133$ and $\sim 120$, respectively. The inset present the X-ray structure of the EcoRI-DNA complex: the grey color structure represents EcoRI, and the yellow color structure represents DNA; the orange balls represent $\mathrm{Cu}^{2+}$ ions. The protein and DNA structures are from a highly refined version ${ }^{28,29}$ of the protein data bank entry $1 \mathrm{CKQ}$. The DNA sequence is TCGCGAATTCGCG. 


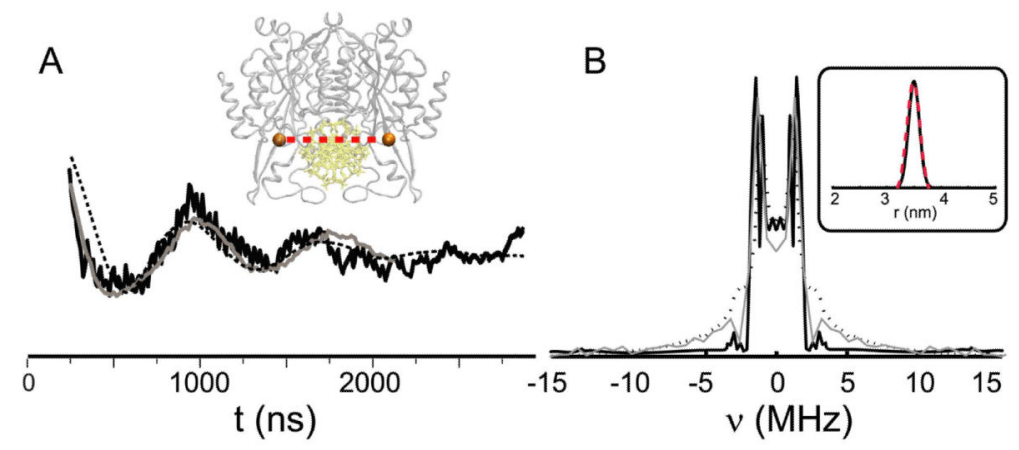

Figure 2.

The comparison of the $\mathrm{Cu}^{2+}$-DEER data (grey solid line) with the experimental (black solid line) $\mathrm{Cu}^{2+}$-DQC data. The simulated DQC trace is shown as a black dotted line. (a) The time traces are shown. The DQC time domain data is obtained by division of a $\pi=16 \mathrm{~ns}$ signal by a $\pi=40 \mathrm{~ns}$ signal. The high frequency component in the DQC data is from proton hyperfine interaction and is not noise. The signal to noise ratio for DEER and DQC were 66 and 61, respectively - details are provided in the Supporting Information. The number of averages for DEER and DQC were 5760 and 1536, respectively. (b) The DEER and DQC spectra are shown and the distance distribution functions are provided in the inset. The distance distribution functions from DEER (red dotted line) and DQC (black solid line) were obtained from fitting of the experimental time domain data. 